\title{
Bias in masked word identification: Unconscious influences of repetition priming
}

\author{
MICHAEL E. J. MASSON \\ University of Victoria, Victoria, British Columbia, Canada
}

\begin{abstract}
The beneficial influence of a prior study episode on subsequent identification of a word includes a large bias component, revealed in the forced-choice variant of the masked word identification test. In that type of test, subjects show a preference for a studied probe over a nonstudied probe, regardless of which one matches the masked target word. The forced-choice test was used in the present experiments to test the possibility that this bias effect is due to conscious recollection. Results show that bias was strongly attenuated (1) by changes in modality between study and test, and (2) under certain conditions, by using a conceptually driven study task. The bias effect was found only when probes were orthographically similar to one another, as predicted by the counter model (Ratcliff \& McKoon, 1997). These results provide strong evidence that the bias effect is not mediated by conscious recollection.
\end{abstract}

The influence of memory for a prior encounter with a stimulus, such as a word, can powerfully affect subsequent encoding of that item. In word identification tests, prior study can both elevate identification accuracy, at least for low-frequency words, and induce a systematic bias in identification decisions (Bowers, 1999; Masson \& MacLeod, 1996; Ratcliff \& McKoon, 1997; Ratcliff, McKoon, \& Verwoerd, 1989; Wagenmakers, Zeelenberg, \& Raaijmakers, 2000). This bias effect is most clearly seen in a forced-choice variant of the masked word identification test in which subjects view a briefly presented target, followed by a mask and then two probe words, one of which matches the target. Bias in responding is apparent when only one of the two probes has been previously studied and subjects show a preference for that item over the nonstudied probe, regardless of which probe actually matches the target. This form of bias results in elevated accuracy when the studied probe matches the target, relative to a baseline condition in which neither probe has been studied, and a comparable reduction in accuracy when the studied probe does not match the target (e.g., Ratcliff \& McKoon, 1997).

As with other influences of prior encoding episodes on identification tests, questions arise as to whether those influences are unconscious or mediated by conscious recollection. Keane, Verfaellie, Gabrieli, and Wong (2000) recently claimed that bias in the forced-choice test is indeed the product of conscious influences. They reported that

This research was supported by research and equipment grants from the Natural Sciences and Engineering Research Council of Canada. I am grateful to Carrie Hicks for assistance in conducting Experiments 1 and 2 and to Allison Barnes and Janine de la Salle for assistance with Experiment 3. I also thank Jeffrey Bowers, Larry Jacoby, and Eric-Jan Wagenmakers for very helpful comments on earlier versions of this article. Correspondence should be sent to M. E. J. Masson, Department of Psychology, University of Victoria, P. O. Box 3050 STN CSC, Victoria, BC, V8W 3P5 Canada (e-mail: mmasson@uvic.ca). amnesic subjects showed a reliable improvement when the studied probe matched the target, relative to a nonstudied condition, but no reliable reduction in accuracy when it was the foil probe that had been studied. In contrast, normal control subjects showed the roughly symmetrical benefit and cost pattern seen with young adults in prior studies. Keane et al. ascribed that symmetrical bias effect to conscious recollection. An alternative interpretation, however, is that the bias effect among control subjects was not mediated by conscious recollection and that for amnesic subjects items used in the experiment might have been experienced as low-frequency words. Low-frequency words show improved accuracy in addition to a possible bias effect in a forced-choice test (Bowers, 1999; Wagenmakers et al., 2000). Furthermore, on trials in which target words were not accurately perceived, amnesic subjects may have adopted the strategy of comparing letter features obtained from the target display with the letter features of the probes, rather than encoding the probes as words. This strategy would mitigate against finding any bias effect that depends on processes that operate at the word level (Bowers, 1999, p. 590).

The strongest empirical evidence in favor of bias arising from unconscious influences of memory was provided by Ratcliff and McKoon (1997). First, the bias effect was maintained even when subjects responded under a short deadline, which was assumed to prevent the use of any strategy involving conscious recollection. Second, they showed that the bias effect was obtained when orthographically similar probe pairs (e.g., died-lied) were used, but not when dissimilar probe pairs were used. Had subjects been using a conscious recollection strategy, it would be expected that the strategy would have affected performance both when similar and when dissimilar probes were used. When subjects were instructed in a manner that invited the use of conscious recollection, both types of probes led to a bias effect (McKoon \& Ratcliff, 2001). In 
the Ratcliff and McKoon (1997) counter model of bias effects, it is assumed that during word identification, the lexical entry for a studied word attracts perceptual evidence that otherwise might have been taken by the entry for the nonstudied probe, thereby biasing the accumulation of evidence in favor of the studied probe. The counter model stipulates that a studied item does not attract evidence counts away from dissimilar items and therefore makes the important prediction that the bias effect will not appear when probe items are orthographically dissimilar (see Schooler, Shiffrin, \& Raaijmakers, 2001, for an alternative model that makes a comparable prediction).

Another means of checking for the involvement of conscious recollection is to attempt to establish dissociations between tests. Two potential dissociations of this kind were examined in the experiments reported here. The first involved modality-specific effects. Tests that primarily depend on conscious recollection of an item's prior occurrence show little sensitivity to changes in modality across study and test, whereas tests that involve target identification without the requirement of conscious recollection are quite sensitive to changes in modality (e.g., Rajaram \& Roediger, 1993). In the case of masked word identification, Clarke and Morton (1983), Jacoby and Dallas (1981), Kirsner, Milech, and Standen (1983), Levy and Kirsner (1989), and Weldon (1991) all obtained more repetition priming when words were studied visually than when they were studied auditorily. The forced-choice variant of masked word identification in question here presumably expresses the influence of prior study through the same mechanism as the standard naming version used in the experiments in which modality effects have been found (see Ratcliff \& McKoon, 1997). Thus, in Experiment 1, it was tested whether the bias effect in the forcedchoice task is modality specific.

The second dissociation was patterned after Jacoby's (1983) demonstration that generating a target item without direct perceptual experience produces little if any benefit on a masked word identification test, although it yields high levels of conscious recollection. Jacoby obtained the opposite pattern with an encoding task that required subjects simply to read study items (see also Weldon, 1991). This approach was used in Experiments 2 and 3 , in which read and generate encoding tasks and their respective influence on bias in the forced-choice test were compared.

\section{EXPERIMENT 1}

Two variants of the masked word identification test were used in Experiment 1. The first version was the standard naming task, in which the subjects were asked to name a briefly presented target. The second was the forced-choice version, in which presentation of a masked target was followed by two orthographically similar probes and the task was to select the probe that matched the target. The critical manipulation was the modality in which the study words were presented: visual versus au- ditory. If repetition priming in these two tests is not entirely reliant on conscious recollection, priming should be sensitive to modality changes in both cases.

\section{Method}

Subjects. The subjects tested in these experiments were students at the University of Victoria who received extra credit in an introductory psychology course for their participation and were native speakers of English. One hundred thirty-two subjects were tested in Experiment 1; 36 were given the naming test, and 96 were given the forced-choice test. Half of the subjects in each test condition were randomly assigned to each of the study modality conditions, visual and auditory.

Materials. The set of critical items consisted of 75 pairs of orthographically similar words. Members of a pair were of the same letter length (4-6 letters) and differed by only one interior letter (e.g., cage, cave). The frequency of occurrence of these words in the English language ranged from 0 to 2,244 per million, with a median frequency of 34 (Kučera \& Francis, 1967). The pairs were arranged as three sets of 25 pairs each for counterbalanced assignment to three study conditions: target, similar, and nonstudied. For pairs assigned to the target condition, the target member of the pair was presented in the study phase; for pairs assigned to the similar condition, the other member of the pair was presented in the study phase; for pairs assigned to the nonstudied condition, neither item was presented at study. Assignment of one member of each pair to serve as the target item for that pair was also counterbalanced across subjects.

Procedure. Instructions and stimuli were presented in 12-point Courier font on a Macintosh microcomputer equipped with two monochrome monitors. One monitor was viewed by the subject, and the other was viewed by the experimenter to allow for on-line scoring of responses. A four-letter word subtended a horizontal visual angle of $1.4^{\circ}$ when viewed from $40 \mathrm{~cm}$. Auditory recordings of study lists were presented via headphones on a Sanyo tape player. A sixkey response box allowed the subject to initiate the trials.

In the study phase, the subjects were told that the purpose of the experiment was to examine how people access their knowledge about words. The subjects were presented with 4 filler items, 50 critical items in random order, and then 4 more filler items. In the visual study condition, each word appeared in the center of the monitor, and the task was to read the word aloud. After the response had been made, the experimenter pressed a key to erase the word. Following a 500-msec pause, the next word appeared. For subjects in the auditory study condition, a word was presented every $2 \mathrm{sec}$ and was repeated aloud by the subject.

The subjects were then presented a series of trials on which a target word was briefly displayed. They were told that the task was to identify each word. In the first part of this test phase, up to 48 trials were presented during which the visual contrast of the briefly presented target word was adjusted to bring each subject's accuracy to a designated level (.60 for the naming test and .75 for the forcedchoice test). The pool of words used for this procedure was different from the filler words used in the study phase and from the critical words. The contrast level reached at the end of this procedure was used in the subsequent test phase. In the Macintosh system used here, contrast levels varied on a scale from 0 (full contrast) to 255 (no contrast). The mean contrast level set for the subjects tested in this naming version of the masked identification test was 68 . For the forced-choice test, the mean contrast setting was 106 .

On each trial of the adjustment procedure, the word READY appeared at the center of the monitor until the subject pressed a key. After a 255-msec blank interval, two hyphens separated by enough spaces to fit the upcoming target word between them then appeared. Following a pause of $255 \mathrm{msec}$, the target word then appeared in lowercase letters between the hyphens. This display was replaced after $45 \mathrm{msec}$ by a mask consisting of a row of ampersands for 
150 msec. The mask was erased and replaced by a row of question marks in the naming test or by two probe words presented side by side in lowercase letters in the forced-choice test. The subject responded either by attempting to name the target or by choosing one of the probe words. In the latter case, the subject pressed either the left or the right key on the response box to select the left or the right probe, respectively.

The test phase consisted of 75 critical trials, presented in the same manner as in the adjustment procedure. The target word on each trial was taken from one of the 75 critical word pairs. In the forced-choice test, the probes presented on a test trial were the target and its orthographically similar partner. For half of the subjects who were given the forced-choice test, the test phase was followed by a recognition memory test in which the subjects were given a printed list of the 75 critical words that had been presented as targets in the test phase. The subjects were instructed to circle any word that had been presented in the study phase. Thus, only the 25 items in the targetstudied condition were valid targets for this test; the remaining 50 items were foils.

\section{Results and Discussion}

The mean proportions of correct responses in the two versions of the masked word identification test and the mean proportion of yes responses in the recognition test are shown in Figure 1. The naming version of the masked word identification test replicated earlier demonstrations of modality specificity in repetition priming on this task (e.g., Jacoby \& Dallas, 1981; Kirsner et al., 1983). As indicated in the figure, auditory study led to only a very small, nonsignificant improvement in performance, whereas visual study produced a robust improvement. ${ }^{1}$ Moreover, an analysis of variance (ANOVA) with study modality and study condition (target, similar, nonstudied) as factors yielded an interaction between study modality and study condition $\left[F(2,68)=4.02, M S_{\mathrm{e}}=0.010, p<\right.$ .05 (as for all statistical tests reported in this article)], indicating that the priming effect was reliably larger in the visual group.

In the forced-choice test, a bias effect similar to that obtained by Ratcliff et al. (1989) and Ratcliff and McKoon (1997) was found. That is, accuracy was higher in the targetstudied condition than in the similar-studied condition, with performance on nonstudied items falling approximately midway between those two conditions. Although it is apparent from Figure 1 that the bias effect was significant in the auditory group as well as in the visual group, the effect was much larger for the visual group, as is indicated by a significant interaction between study modality and study condition in an ANOVA with these two factors $\left[F(2,188)=5.26, M S_{\mathrm{e}}=0.006\right]$. As with the naming version of masked word identification, the forced-choice version was sensitive to changes in modality between study and test.

In contrast to the modality-specific effects seen in the two versions of the masked word identification test, the recognition test resulted in very similar performances for the two modality groups: An ANOVA with study modality and study condition as factors failed to produce a significant interaction $(F<1)$, although the subjects were much more likely to respond "yes" to studied targets or to

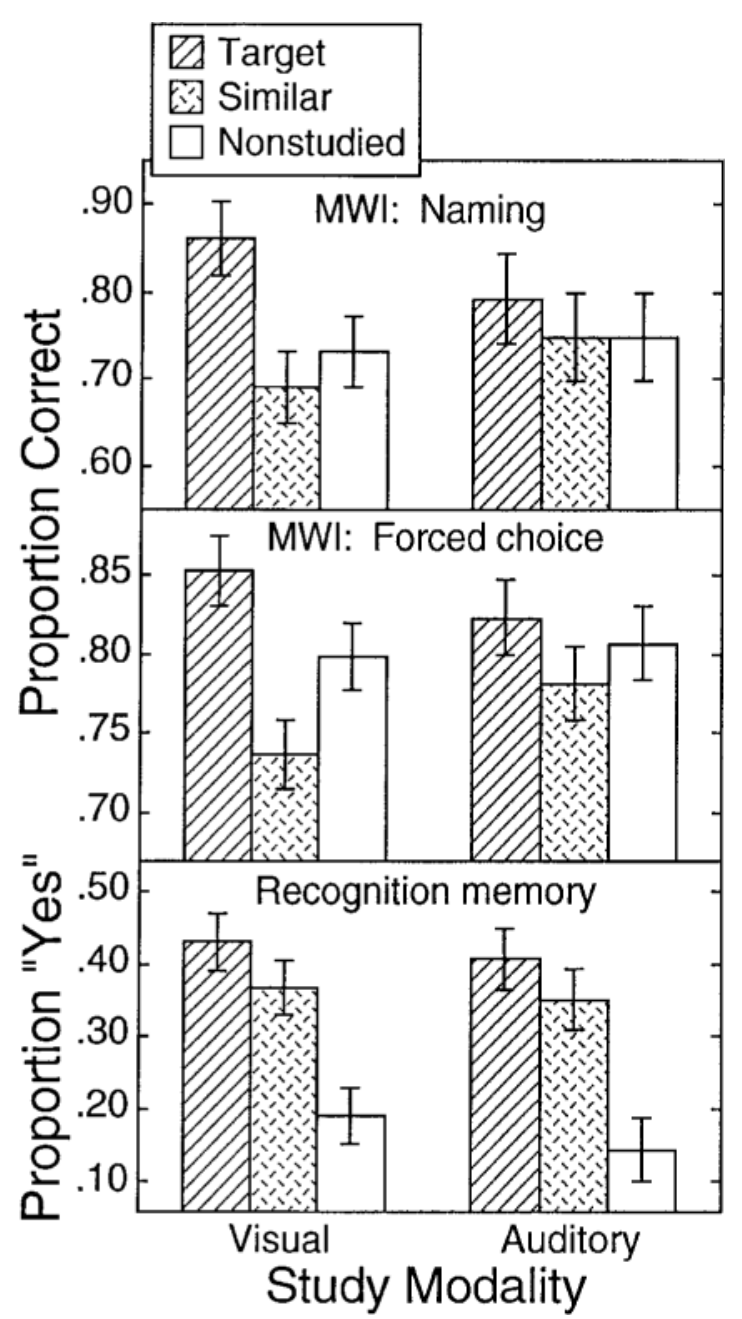

Figure 1. Mean proportion of correct responses on naming and forced-choice versions of the masked word identification (MWI) test and mean proportion of yes responses on the recognition memory test in Experiment 1. Error bars represent $95 \%$ withinsubjects confidence intervals (Loftus \& Masson, 1994) based on all conditions within a modality.

items that were orthographically similar to studied targets than to nonstudied items. Although a change in modality did not entirely eliminate the bias effect in the forcedchoice test, it did result in a large reduction. Combined with the lack of modality effect in the recognition test, this outcome supports the conclusion that conscious recollection is not entirely, although it may be partly, responsible for the bias effect.

\section{EXPERIMENT 2}

In the second experiment, a different encoding manipulation was applied. The subjects encoded words either by reading them or by generating them from a semantic cue without actually seeing them. In contrast to the effect of a read task on later identification performance, some gen- 
eration tasks yield relatively little or no priming (e.g., Jacoby, 1983; Weldon, 1991). This pattern of performance is opposite to that seen with tests of conscious recollection whereby generation leads to much better performance than does a read task.

An exception to this pattern, however, is that Masson and MacLeod (1992; MacLeod \& Masson, 1997) have shown that generation encoding can produce as much repetition priming as reading when masked word identification is used at test. This priming might reflect the contribution of conceptual processes to identification tests or the visualization of a generated target's orthography at the time of encoding (Masson \& MacLeod, 1992, 2002). It is not clear whether these influences would operate in the forced-choice identification test, but if they do, it would spoil the attempt to establish the hypothesized dissociation because both read and generate encoding tasks would lead to a bias effect. Therefore, another manipulation included in Experiment 2 was whether probes were orthographically similar or dissimilar. If conscious recollection induces the bias effect, it should be seen for both types of probe pairs.

\section{Method}

Subjects. Forty subjects were tested, half in the similar-probe group and half in the dissimilar-probe group.

Materials. The critical items consisted of 120 pairs of orthographically similar words defined as in Experiment 1 . These words ranged in frequency from 0 to 2,244 per million, with median frequency of 27 (Kučera \& Francis, 1967). A generation cue was created for each of these 240 words, consisting of a phrase and the first letter of the critical word (e.g., the cue for core was the center of an apple - c). For subjects in the dissimilar-probes condition, the pairs of critical words were re-paired so that the two words of each new pair were of equal length but they were not orthographically similar (e.g., core, ship). Each of these two versions of 120 critical word pairs was arranged into five sets of 24 pairs for counterbalanced assignment to the five study conditions: read-target, read-foil, generatetarget, generate-foil, and nonstudied. One member of each pair in the read-target and generate-target conditions was read or generated at study and then appeared as a target in the test phase. For pairs in the read-foil and generate-foil conditions, one member of each pair was read or generated at study but its partner appeared as the target in the test phase. For items in the nonstudied condition, neither member of the pair was presented at study. Assignment of one member of each pair to serve as the target item for that pair was counterbalanced across subjects.

Procedure. The procedure and instructions were similar to those of Experiment 1, except that in the study phase, only visual presentation of items was used. Four practice items were presented, followed by 96 critical items ( 48 read and 48 generate) in random order. Each item in the read condition appeared at the center of the computer monitor until the subject read it aloud. For items in the generate condition, a generate cue was presented for a maximum of $10 \mathrm{sec}$ or until the subject produced a word in response to it; accuracy of these responses was recorded by the experimenter. If the subject failed to generate the correct response word, the experimenter read it aloud to the subject.

The forced-choice version of the masked word identification test was used in the test phase, following the same procedure as in Experiment 1 , including the adjustment procedure to set individual contrast levels to achieve accuracy of .75 in the nonstudied condition. The adjustment procedure used probe pairs appropriate for the similarity condition to which the subject had been assigned. The subjects in the similar-probe group were presented probe pairs that were orthographically similar, and the subjects in the dissimilarprobe group were presented orthographically dissimilar probes. The exposure duration of targets was $30 \mathrm{msec}$. The mean contrast level for the similar-probe group was 122 , and the mean for the dissimilarprobe group was 92 . There were 120 critical trials.

\section{Results and Discussion}

The mean proportion of correctly generated targets during study was .89 . The mean proportions of correct responses in the test phase are shown in Figure 2. The data for the generate condition were not conditionalized on correct generation at study, but the conditionalized data show the same pattern as that reported here. The subjects in the similar-probe group clearly demonstrated the usual bias effect both for the read condition and the generate condition. An ANOVA (excluding data from the nonstudied condition) with study task (read and generate) and study condition (target and foil) yielded no interaction $(F<1)$, indicating that the bias effect was equally strong for both encoding tasks. In the dissimilar-probe group, no bias effects were evident. The power to detect in this group a bias effect of the magnitude found in the similar-probe group, averaging across read and generate conditions, was estimated to be .99 .

The read and generate encoding tasks produced nearly identical bias effects in the forced-choice test, but these effects were seen only when probes were orthographically

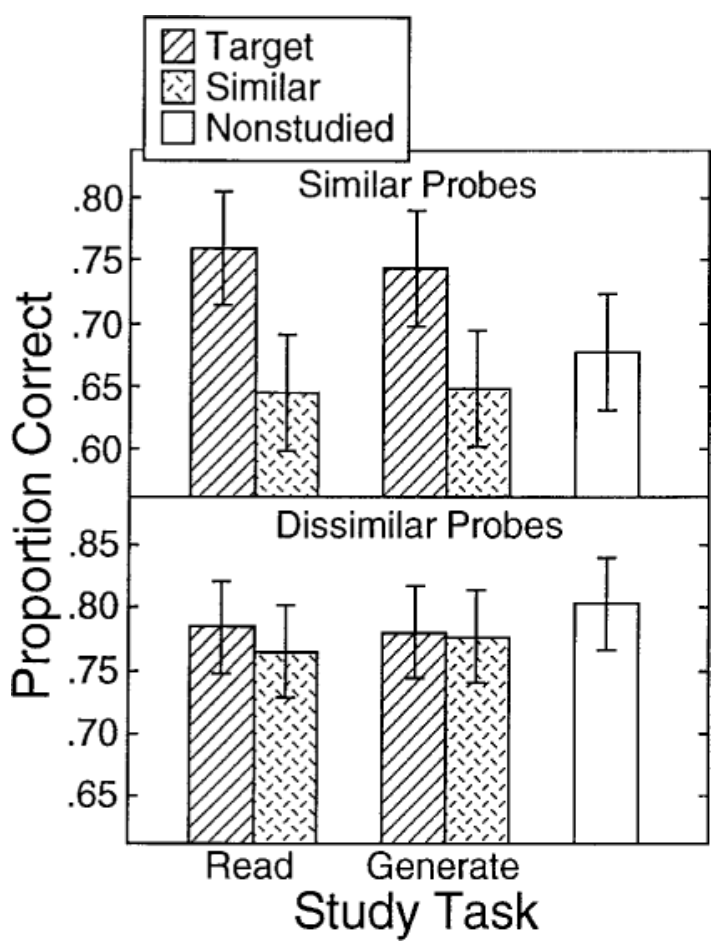

Figure 2. Mean proportion of correct responses on the masked word identification task for the orthographically similar- and dissimilar-probe groups in Experiment 2. Error bars represent 95\% within-subjects confidence intervals for all conditions within a probe similarity condition. 
similar. This pattern of results supports the conclusion that the observed bias was not the product of conscious recollection of previously studied items, but fails to establish the dissociation that was originally sought, whereby bias would not have appeared for generate items.

\section{EXPERIMENT 3}

It might be argued that the occurrence of a bias effect only among those subjects who were presented orthographically similar probes was a result of strategic processes. Specifically, the subjects shown only dissimilar probes might have looked for distinguishing letter features in the target display (Bowers, 1999). When probes are dissimilar, this strategy could be effective and might be very different from the approach taken by subjects tested with orthographically similar probes. Therefore, in Experiment 3, a replication of Experiment 2 was carried out in which probe similarity was manipulated within subjects so that from trial to trial, the subjects would be unaware of whether the probes following a particular target were orthographically similar or not. If the bias effect continues to be restricted to similar-probe trials under these conditions, the strategic account would be much less plausible.

\section{Method}

Subjects. Twenty-four subjects were tested.

Materials. The 120 critical items and generation cues from Experiment 2 were used. As in that experiment, two versions of the word pairs were constructed, one with orthographically similar pairs and one with dissimilar pairs. Each of these versions was divided into eight lists of 15 items for counterbalanced assignment to one of eight conditions created by a factorial combination of encoding task (read and generate), study condition (target and foil), and probe similarity (similar and dissimilar). A set of 240 filler words similar to the critical items in length and distribution of frequency in the language was created. These items were arranged as 60 orthographically similar pairs and 60 orthographically dissimilar pairs. As with the critical items, words within a pair were of the same length. These filler items were used as nonstudied items in the test phase of the experiment. By using filler items in the nonstudied condition we avoided the requirement of creating highly reliable generation cues for these items, but sacrificed the opportunity to compare performance in the nonstudied condition with the target- and foil-studied conditions. That comparison is useful if one is interested in assessing the influence of prior exposure on accuracy (e.g., Ratcliff et al., 1989; Wagenmakers et al., 2000), but that was not our goal here. Another set of 60 word pairs, 30 orthographically similar and 30 dissimilar, was created for use in the adjustment procedure to establish individual contrast levels.

Procedure. The procedure and instructions were similar to those of Experiment 2, except that in the study phase, 120 critical trials (60 read and 60 generate) were presented, with two filler trials preceding and another two filler trials following. The adjustment procedure included both orthographically similar and dissimilar pairs but, to simplify the program that controlled the procedure, adjustments were based only on performance on the similar pairs. A fixed value of 70 was added to the resulting contrast level and was applied for all subjects on trials involving orthographically dissimilar pairs. On the Macintosh contrast scale of 0 (full contrast) to 255 (no contrast), the mean contrast level set for the subjects based on orthographically similar probes was 111 . In adding 70 to each subject's contrast value (thereby reducing the contrast level) for orthographically dis- similar probes, the maximum value allowed was 210 . In the test phase, the subjects were presented 120 critical trials (constituting target- and foil-studied conditions) and 120 filler trials (constituting nonstudied conditions) in random order, with half of the trials of each type using orthographically similar probes, and half using dissimilar probes.

\section{Results and Discussion}

The subjects correctly generated a mean proportion of .90 targets in the generate task during the study phase. The mean proportions of correct responses in the test phase (not conditionalized on correct responding in the study phase) are presented in Figure 3 for the target- and foilstudied conditions. Data for the nonstudied condition are not shown there because filler items were used in this condition and direct comparison with the two studied conditions would not be appropriate. Mean proportion correct on nonstudied items was .78 in the similar-probe condition and .73 in the dissimilar-probe condition. The data for target- and foil-studied conditions in Figure 3 indicate a trend toward an advantage in the similar-probe condition. This trend indicates only that our adjustment of contrast values for the dissimilar condition was a bit too extreme. More important, comparison of the target- and foil-studied conditions show that the results of Experi-

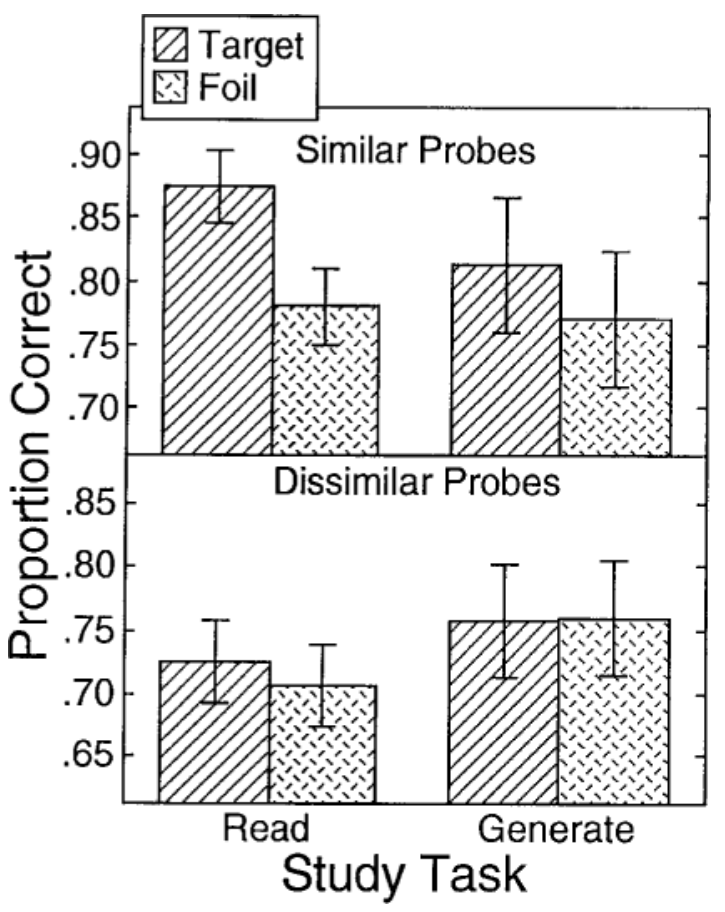

Figure 3. Mean proportion of correct responses on the masked word identification task for the orthographically similar- and dissimilar-probe groups in Experiment 2. Data for the nonstudied condition are not shown because filler items were used in this condition and direct comparison with the two studied conditions would not be appropriate. Error bars represent $95 \%$ withinsubjects confidence intervals for pairs of target- and foil-studied conditions. 
ment 2 were replicated for items in the read condition, in which a clear bias effect was found for orthographically similar but not for dissimilar probes. ${ }^{2}$ In the generate condition, however, no bias effect was seen even with orthographically similar probes. The power to detect an effect in the generate condition for orthographically similar probes, based on the effect size seen in the read condition and a directional test, was .86.

The manipulation of probe similarity within subjects did little to affect the outcome when the study task involved reading target words. Clearly, the possibility that a strategic influence was responsible for the modulation of the bias effect by probe similarity seems unlikely given this outcome, at least for the read condition. The lack of evidence for a bias effect for the generate condition is surprising, given the outcome of Experiment 2. One possibility is that conscious recollection was responsible for the bias effect seen in that experiment for items studied in the generate condition. That notion seems doubtful, given that there was no tendency for the subjects to show a bias effect following generate encoding when probes were dissimilar. An alternative explanation is that although the subjects in Experiment 3 were tempted to prefer probes that had been generated at study (perhaps because of enhanced processing fluency), they may have recollected that earlier encoding event, leading them to discount the current experience by correctly attributing it to the study episode (e.g., Huber, Shiffrin, Lyle, \& Ruys, 2001; Jacoby $\&$ Whitehouse, 1989). The rather wide confidence intervals for the generate condition seen in Figure 3, reflecting the occurrence of some strong reversals in the difference between target- and foil-studied conditions, is consistent with this hypothesis. It is not clear why discounting apparently failed to operate in Experiment 2. The two primary differences between Experiments 2 and 3 were the use of a higher proportion of nonstudied items and a within-subjects manipulation of probe similarity in Experiment 3. Either or both factors could be responsible.

In any case, there is strong evidence in this pattern of results to support the conclusion that the bias effect seen in the read condition was not due to conscious recollection. Many previous studies have shown that, under conditions very much like those in effect here, conscious recollection following a generate study task is far superior to recollection following a read task (Jacoby, 1983; MacLeod \& Masson, 1997; Masson \& MacLeod, 1992; Slamecka \& Graf, 1978). Despite that difference, it was the read task that consistently yielded a bias effect. The present results indicate that conscious recollection is not sufficient to sustain the bias effect seen in the forced-choice version of masked word identification.

\section{CONCLUSION}

The present experiments produced two new dissociations between bias in the forced-choice test and conscious recollection: (1) The bias effect was sensitive to changes in modality between study and test, whereas recognition memory was not, and (2) when probe similarity was manipulated within subjects, a bias effect was obtained for read but not for generate items. The latter effect contrasts with the fact that conscious recollection is known to be much better for generate items. Taken together with the finding that no bias effect emerged when probes were orthographically dissimilar, these results strongly support the conclusion that bias in the forced-choice task is driven by unconscious influences of memory for prior experience. These results are not consistent with the Keane et al. (2000) proposal that such bias effects are the product of conscious recollection.

The present demonstration of modality specificity in the bias effect has implications for both the counter model (Ratcliff \& McKoon, 1997) and the REM model (Schooler et al., 2001) of bias effects. If the counter model is correct, these experiments suggest that the bias induced in lexical entries, or counters, is modality specific. One possible reason for this specificity would be that separate entries exist for visual and auditory modalities (see Clarke \& Morton, 1983, for a similar suggestion). In the REM model, bias arises from the inclusion of contextual information in the lexical representation of studied items. When a test item is flashed, perceptual and contextual features of that event are matched to existing lexical representations. The contextual information included in this match process favors a previously studied probe because its lexical representation contains matching contextual features. If contextual features are responsible for the bias effect, the results of Experiment 1 suggest that these features are in some way modality specific, rather than representing general aspects of the study context.

Finally, the inconsistent pattern of effects of prior study found with the generate encoding task suggests that subjects may discount the influence of prior study when study episodes are easy to recollect. Recent empirical and theoretical work on discounting effects in the forced-choice test (e.g., Huber et al., 2001; Ratcliff \& McKoon, 2001) has initiated a line of investigation that promises to lead to a more detailed understanding of discounting and its relation to conscious and unconscious influences of memory.

\section{REFERENCES}

Bowers, J. S. (1999). Priming is not all bias: Commentary on Ratcliff and McKoon (1997). Psychological Review, 106, 582-596.

Clarke, R, \& Morton, J. (1983). Cross modality facilitation in tachistoscopic word recognition. Quarterly Journal of Experimental Psychology, 35A, 79-96.

Huber, D. E., Shiffrin, R. M., Ly Le, K. B., \& Ruys, K. I. (2001). Perception and preference in short-term word priming. Psychological Review, 108, 149-182.

JACOBY, L. L. (1983). Remembering the data: Analyzing interactive processes in reading. Journal of Verbal Learning \& Verbal Behavior, 22, 485-508.

JACOBY, L. L., \& DALlas, M. (1981). On the relationship between autobiographical memory and perceptual learning. Journal of Experimental Psychology: General, 110, 306-340.

JACOBY, L. L., \& Whitehouse, K. (1989). An illusion of memory: False recognition influenced by unconscious perception. Journal of Experimental Psychology: General, 118, 126-135. 
Keane, M. M., Verfaellie, M., Gabrieli, J. D. E., \& Wong, B. M. (2000). Bias effects in perceptual identification: A neuropsychological investigation of the role of explicit memory. Journal of Memory \& Language, 43, 316-334.

Kirsner, K., Milech, D., \& Standen, P. (1983). Common and modalityspecific processes in the mental lexicon. Memory \& Cognition, 11, 621-630.

KuČera, H., \& Francis, W. (1967). Computationalanalysis of presentday American English. Providence, RI: Brown University Press.

Levy, B. A., \& KirSner, K. (1989). Reprocessing text: Indirect measures of word and message level processes. Journal of Experimental Psychology: Learning, Memory, \& Cognition, 15, 407-417.

Loftus, G. R., \& Masson, M. E. J. (1994). Using confidence intervals in within-subject designs. Psychonomic Bulletin \& Review, 1, 476490.

MacLeod, C. M., \& Masson, M. E. J. (1997). Priming patterns are different in masked word identification and word fragment completion. Journal of Memory \& Language, 36, 461-483.

Masson, M. E. J., \& MacLeod, C. M. (1992). Reenacting the route to interpretation: Enhanced perceptual identification without prior perception. Journal of Experimental Psychology: General, 121, 145-176.

Masson, M. E. J., \& MacLeod, C. M. (1996). Contributions of processing fluency to repetition effects in masked word identification. Canadian Journal of Experimental Psychology, 50, 9-21.

Masson, M. E. J., \& MacLeod, C. M. (2002). Covert operations: Orthographic recoding as a basis for repetition priming in word identification. Journal of Experimental Psychology: Learning, Memory, \& Cognition, 28, 858-871.

McKoon, G., \& Ratcliff, R. (2001). Counter model for word identification: Reply to Bowers (1999). Psychological Review, 108, 674-681.

RAJARAm, S., \& Roediger, H. L., III (1993). Direct comparison of four implicit memory tests. Journal of Experimental Psychology: Learning, Memory, \& Cognition, 19, 765-776.

Ratcliff, R., \& McKoon, G. (1997). A counter model for implicit priming in perceptual word identification. Psychological Review, 104, 319-343.
Ratcliff, R., \& McKoon, G. (2001). A multinomial model for shortterm priming in word identification. Psychological Review, 108, 835846.

Ratcliff, R., McKoon, G., \& Verwoerd, M. (1989). A bias interpretation of facilitation in perceptual identification. Journal of Experimental Psychology: Learning, Memory, \& Cognition, 15, 378-387.

Schooler, L. J., Shiffrin, R. M., \& RaAjmakers, J. G. W. (2001). A Bayesian model for implicit effects in perceptual identification. Psychological Review, 108, 257-272.

SlameCKA, N. J., \& GRAF, P. (1978). The generation effect: Delineation of a phenomenon. Journal of Experimental Psychology: Human Learning \& Memory, 4, 592-604.

Wagenmakers, E.-J. M., Zeelenberg, R., \& RaAijmakers, J. G. W. (2000). Testing the counter model for perceptual identification: Effects of repetition priming and word frequency. Psychonomic Bulletin \& Review, 7, 662-667.

WeLdon, M. S. (1991). Mechanisms underlying priming on perceptual tests. Journal of Experimental Psychology: Learning, Memory, \& Cognition, 17, 526-541.

\section{NOTES}

1. Most of the statistical inferences discussed in this article are based on the $95 \%$ within-subjects confidence intervals presented in the figures. Interaction effects are sometimes reported to test for differences between pairwise effects seen in the figures.

2. It is clear from Experiments 1 and 2 and from the other studies cited here that differences between target- and foil-studied conditions in the forced-choice paradigm are, for the most part, a product of bias. Thus, although the use of filler items in the nonstudied condition precludes an additional demonstration of that fact, the influence of prior study on forced-choice performance in Experiment 3 is assumed to be primarily one of bias.

(Manuscript received May 22, 2000; revision accepted for publication August 14, 2001.) 